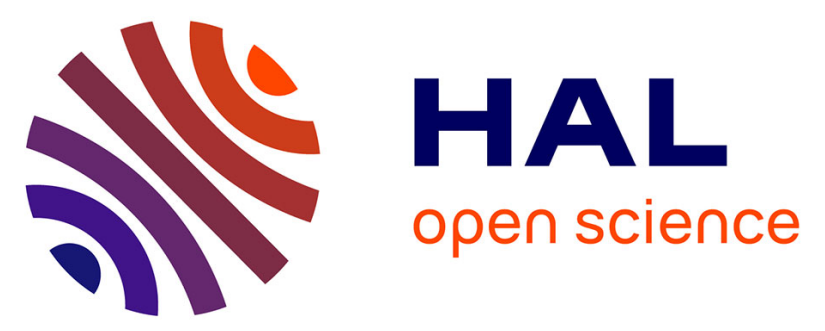

\title{
Generation and manipulation of domain walls using a thermal gradient in a ferrimagnetic TbCo wire
}

R. Tolley, T. Liu, Y. Xu, S. Le Gall, M. Gottwald, Thomas Hauet, M. Hehn, F. Montaigne, E. E. Fullerton, S. Mangin

\section{- To cite this version:}

R. Tolley, T. Liu, Y. Xu, S. Le Gall, M. Gottwald, et al.. Generation and manipulation of domain walls using a thermal gradient in a ferrimagnetic TbCo wire. Applied Physics Letters, 2015, 106 (24), pp.242403. 10.1063/1.4922603 . hal-01246640

\section{HAL Id: hal-01246640 \\ https://hal.science/hal-01246640}

Submitted on 12 Mar 2020

HAL is a multi-disciplinary open access archive for the deposit and dissemination of scientific research documents, whether they are published or not. The documents may come from teaching and research institutions in France or abroad, or from public or private research centers.
L'archive ouverte pluridisciplinaire $\mathbf{H A L}$, est destinée au dépôt et à la diffusion de documents scientifiques de niveau recherche, publiés ou non, émanant des établissements d'enseignement et de recherche français ou étrangers, des laboratoires publics ou privés. 


\section{AIP Appled phy \\ Letters}

\section{Generation and manipulation of domain walls using a thermal gradient in a ferrimagnetic TbCo wire}

R. Tolley, T. Liu, Y. Xu, S. Le Gall, M. Gottwald, T. Hauet, M. Hehn, F. Montaigne, E. E. Fullerton, and S. Mangin

Citation: Applied Physics Letters 106, 242403 (2015); doi: 10.1063/1.4922603

View online: http://dx.doi.org/10.1063/1.4922603

View Table of Contents: http://scitation.aip.org/content/aip/journal/apl/106/24?ver=pdfcov

Published by the AIP Publishing

\section{Articles you may be interested in}

Light-induced magnetization reversal of high-anisotropy TbCo alloy films

Appl. Phys. Lett. 101, 162408 (2012); 10.1063/1.4759109

Modulation of domain wall dynamics in TbFeCo single layer nanowire

J. Appl. Phys. 111, 083921 (2012); 10.1063/1.4704395

Magnetoresistive effects in perpendicularly magnetized Tb-Co alloy based thin films and spin valves

J. Appl. Phys. 111, 083904 (2012); 10.1063/1.3703666

High domain wall magneto-resistance in amorphous TbFeCo wires

Appl. Phys. Lett. 99, 122501 (2011); 10.1063/1.3641428

Enhanced difference in switching fields for perpendicular magnetic spin valves with a composite [ Co / Ni ] N / TbCo reference layer

J. Appl. Phys. 109, 023907 (2011); 10.1063/1.3536476

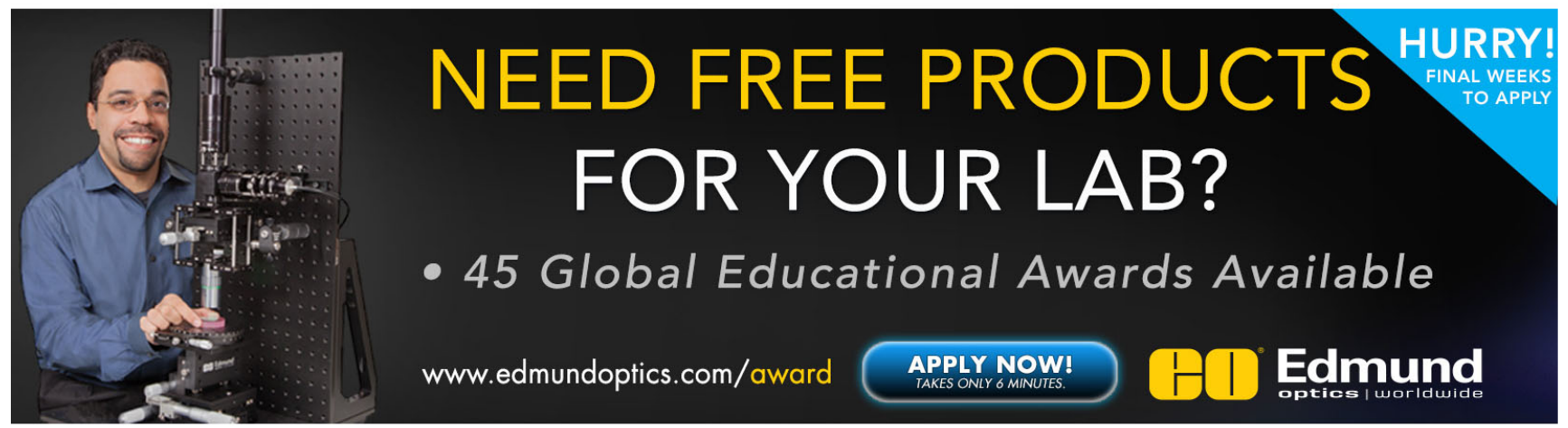




\title{
Generation and manipulation of domain walls using a thermal gradient in a ferrimagnetic $\mathrm{TbCo}$ wire
}

\author{
R. Tolley, ${ }^{1,2}$ T. Liu, ${ }^{1}$ Y. Xu, ${ }^{1}$ S. Le Gall, ${ }^{1, a)}$ M. Gottwald, ${ }^{2, b)}$ T. Hauet, ${ }^{1}$ M. Hehn, ${ }^{1}$ \\ F. Montaigne, ${ }^{1}$ E. E. Fullerton, ${ }^{2}$ and S. Mangin ${ }^{1}$ \\ ${ }^{1}$ Institut Jean Lamour, UMR 7198 CNRS, Universite de Lorraine, Vandoeuvre-les-Nancy, France \\ ${ }^{2}$ Center for Magnetic Recording Research, University of California San Diego, La Jolla, \\ California 92093-0401, USA
}

(Received 18 December 2014; accepted 31 May 2015; published online 15 June 2015)

\begin{abstract}
We demonstrate the ability to create, control the propagation, and annihilate domain walls in 25$\mathrm{nm}$ thick $\mathrm{Tb}_{22} \mathrm{Co}_{78}$ ferrimagnetic alloy wires using a temperature gradient under a constant applied field. The temperature gradient is generated by passing a current through the wire, and the domain wall properties are imaged using Kerr microscopy. The manipulation of the domain wall is made possible by creating a temperature gradient such that the temperature at one end of the wire is above the compensation temperature for the TbCo alloy, while the other end remains below the compensation temperature. By tuning the intensity of the applied magnetic field and the current flowing inside the wire, it is possible to carefully control the domain wall position that can then be stabilized under zero applied field and current. (C) 2015 AIP Publishing LLC.

[http://dx.doi.org/10.1063/1.4922603]
\end{abstract}

The ability to manipulate magnetic domain walls (DWs) has attracted interest for both fundamental understandings as well as future applications. Moving magnetic DWs are desired for permanent data storage and magnetic memory applications ${ }^{1}$ as well as magnetic logic. ${ }^{2}$ The potential exists for race track memories ${ }^{1}$ that are designed to store and transfer information in non-mechanical, high-density, low-power, and non-volatile recording systems. To this end, numerous fundamental studies have been conducted to find new means to manipulate magnetic domain walls. It is well known that a magnetic field applied to a magnetic material can lead to domain wall formation and propagation. The possibility of reversing magnetization using a polarized current was theoretically predicted over 30 years ago by Freitas and Berger, ${ }^{3}$ but is still under investigation. ${ }^{4}$ Even, more recently, the possibility using of strain, ${ }^{5}$ electric field, ${ }^{6}$ optical fields, ${ }^{7}$ or polarized pulse laser $^{8-10}$ to reverse magnetization has attracted much interest. Heat itself has recently been proposed $^{11}$ as an efficient control parameter for spin manipulation, where a temperature gradient can generate spin current due to electron and/or magnon flow. This thermoelectric phenomenon of spin current generation is known as the spin Seebeck effect and has also been suggested ${ }^{12}$ as a control parameter for DW manipulation. Hrabec et al. have shown that using a composition gradient it is possible to generate a DW in GdCo ferrimagnetic alloys, under a magnetic field, in the vicinity of the compensation composition. ${ }^{13}$ Ferrimagnetic alloy materials are studied recently because of their suitability for optical switching, ${ }^{7,8}$ positive and negative exchange bias, ${ }^{14,15}$ negative GMR effects, ${ }^{16,17}$ efficient current induced domain wall motion, ${ }^{18}$ and tunable Curie temperature. ${ }^{19}$ In this letter, we examine how we can nucleate, propagate, and then annihilate $\mathrm{DWs}^{20,21}$ in a ferrimagnetic

\footnotetext{
${ }^{a)}$ Current address: Génie Electrique et Electronique de Paris, UMR-CNRS 8507, CentraleSupelec, Univ. Paris-Sud XI, F-91192 Gif-sur-Yvette Cedex, France.

b) Matthias Gottwald is currently affiliated with Qualcomm.
}

$\mathrm{Tb}_{22} \mathrm{Co}_{78}$ via a thermal gradient under applied magnetic fields.

The $\mathrm{Ta}(3 \mathrm{~nm}) / \mathrm{Tb}_{22} \mathrm{Co}_{78}(25 \mathrm{~nm}) / \mathrm{Ta}(5 \mathrm{~nm})$ thin-film was grown by dc magnetron sputtering onto a silicon substrate, where $\mathrm{TbCo}$ is amorphous and has strong perpendicular magnetic anisotropy (PMA). ${ }^{17}$ In this sample, the magnetization of the $\mathrm{Co}$ and $\mathrm{Tb}$ sublattices is exchange coupled antiferromagnetically, and each sublattice has a differing temperature dependence of magnetization. Depending on the $\mathrm{Tb}_{1-\mathrm{x}} \mathrm{Co}_{\mathrm{x}}$ composition, three cases are possible. For Co composition, $\mathrm{x}>0.85$ and $\mathrm{x}<0.75$, the net magnetization is parallel or antiparallel to the Co sublattice at all temperatures. ${ }^{19}$ For intermediate alloy compositions $(0.75<\mathrm{x}<0.85)$, the differing dependence on temperature leads to the existence of a magnetic compensation temperature $\left(\mathrm{T}_{\text {comp }}\right)$, where the two sublattices are equal in magnitude and opposite in direction. In this case, for temperatures above $T_{\text {comp }}$, the net magnetization of the film is parallel to the magnetization of the Co sublattice (i.e., Co dominant); while below $\mathrm{T}_{\text {comp }}$, the net magnetization is parallel to the $\mathrm{Tb}$ sublattice ( $\mathrm{Tb}$ dominant). Magnetization of our full film sample was measured by SQUID VSM and displays a compensation temperature of $375 \mathrm{~K}$ in agreement with Ref. 19.

It is possible to exploit the compensation temperature to create, controllably propagate, and annihilate DWs as shown schematically in Fig. 1. This is achieved by applying a temperature gradient to a film, where the temperature range spans either side of $\mathrm{T}_{\text {comp. }}$. At the compensation temperature, the sublattice moments are equal in magnitude and aligned in opposite directions. This produces a vanishing magnetic moment at the compensation point that increases on either side. When under an applied field, this leads to 3 regions of the magnetic film as shown in Fig. 1. In the regions where the temperature is higher or lower than the compensation temperature, the net magnetization of the alloy is along the $\mathrm{Co}$ or $\mathrm{Tb}$ sublattice magnetization, respectively (Fig. 1). So, if one could apply an infinite magnetic field perpendicular to 


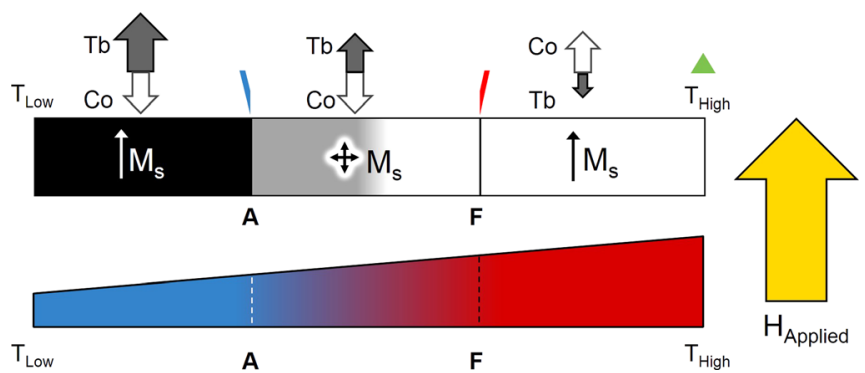

FIG. 1. Sketch of the magnetic configuration inside the wire when a field is applied perpendicular to the wire, and a temperature gradient is present along the wire. The gradient is applied such that the temperature is high on the right and low on the left with the compensation temperature reached halfway. The dominant sublattice magnetization will align itself in the direction of the magnetic field, where the Cobalt (resp. Terbium) is in shown in white (resp. black) and is shown by the color of the film. In the grey gradient intermediate region, the orientation of the magnetization depends on the motion of the gradient (cooling or heating), as neither sublattice is sufficiently dominant to control the net magnetization.

the film, the net magnetization everywhere in the wire would align along the field. The Co sublattice magnetizations in the Co-dominant region (area in white in Fig. 1) and in the Tb-dominant region (area in black in Fig. 1) would then be anti-parallel, and a Co and $\mathrm{Tb}$ domain wall would be localized at the position where the temperature reaches $\mathrm{T}_{\text {comp }}$. However, due to finite field limitations, a hysteretic effect is observed as the net magnetization needs to reach a certain value before the sample anisotropy can be overcome. In this general picture, an indeterminate area not affected by the field can be defined (gradient grey area).

To experimentally explore the DW generation and manipulation, the $\mathrm{Tb}_{22} \mathrm{Co}_{78}$ sample was annealed at $200^{\circ} \mathrm{C}$ for $2 \mathrm{~h}$ and then patterned into a series of wires with Hall crosses, from $2 \mu \mathrm{m}$ to $10 \mu \mathrm{m}$ wide and $20 \mu \mathrm{m}$ to $40 \mu \mathrm{m}$ long by electron-beam lithography and gold contacts were deposited to electrically connect the sample. To observe the DW propagation, we both heat the sample and create a temperature gradient. Joule heating was used by injecting a current through the contacts into the wire as shown in Fig. 2(a)). An engineered defect (shown by the green triangle in Fig. 2) was introduced on the wire, which serves to increase the local current density and thus the local temperature-forming a hot spot. As a counterpart to the hot spot is an expansion pad (left side) - a region where the width of the wire increases, and consequently the temperature decreasesserving as a heat sink. A temperature gradient is, therefore, present along the wire and can be tuned by changing the intensity of the injected current. Note that the current was injected in both directions in order to study possible spin transfer torque effects as has been proposed. ${ }^{18}$ However, the DW propagation results are independent of current direction. It was suggested that spin torque efficiency should be enhanced in the vicinity of compensation, but such effects were not observed-in agreement with the work reported on GdCo. ${ }^{13}$

By injecting a large enough current, the temperature of the "hot spot" can rise above the compensation temperature, while the expansion pad remains cooler than the compensation temperature. In that case, for a large enough field applied along the anisotropy axis (perpendicular to the film

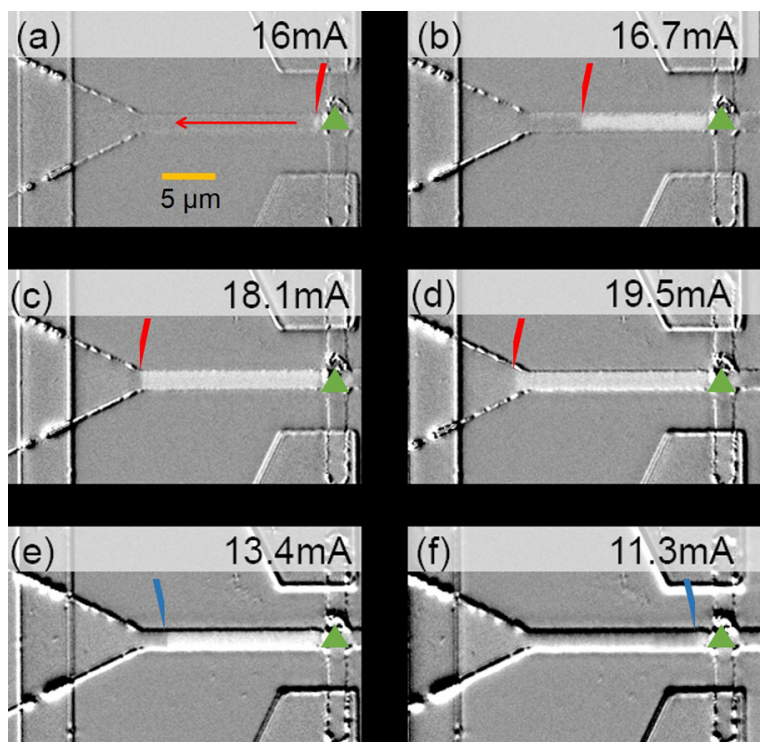

FIG. 2. Evolution of the magnetic configuration along a $2 \mu \mathrm{m}$ TbCo wire measured under an applied field of $350 \mathrm{mT}$ using a Kerr microscope and observed as a function of the injected current intensity. (a) A $16 \mathrm{~mA}$ current is injected (red arrow), and a domain is nucleated at the hot spot localized on the defect (green triangle). As we move away from the right defect toward the expansion pad contact on the left, the temperature decreases, creating a temperature gradient along the wire ((a)-(d)). Increasing the current causes the temperature to increase, and the domain wall is shown to move toward the expansion pad-following the thermal gradient motion. ((e) and (f)) When the current is sufficiently reduced, the domain wall will retract back to the nucleation site. When the Co dominant domain expands, the position of the domain wall is marked by a red sign; whereas when the Co dominant domain retracts, the position of the domain wall is marked by a blue sign. The distance between the engineered defect and the pad is $20 \mu \mathrm{m}$. Current densities required to propagate are between $2 \times 10^{11} \mathrm{~A} / \mathrm{m}^{2}$ and $3 \times 10^{11} \mathrm{~A} / \mathrm{m}^{2}$

plane), a DW nucleates. Figure 2 shows that for $350 \mathrm{mT}$ when a current of $16 \mathrm{~mA}\left(2.4 \times 10^{11} \mathrm{~A} / \mathrm{m}^{2}\right)$ is injected, the temperature close to the nucleation site is sufficiently larger than the compensation temperature, so that a Co-dominant domain can switch to align with the field and consequently nucleate a domain wall. By increasing the dissipated power through the wire, the Co-dominant domain can be seen to propagate across the film as seen in Figs. 2(a)-2(d). As the power is increased, the domain propagates towards the expansion pad at the far left of the sample-where an increased area reduces heating. The domain can reach the expansion pad and can start to enter the pad for current of $19.5 \mathrm{~mA}$ (around $3 \times 10^{11} \mathrm{~A} / \mathrm{m}^{2}$ ). When the current is reduced, the process is not smoothly reversible. The current needs to be decreased to $13.5 \mathrm{~mA}\left(2 \times 10^{11} \mathrm{~A} / \mathrm{m}^{2}\right)$ to observe the domain wall retracting. This hysteretic behavior observed between the formation and annihilation currents highlights a difference in the switching temperatures of the system.

From a series of measurements similar to that shown in Fig. 2, four different events may be defined, which happen for different sets of fields and currents ( $\mathrm{I}$ and $\mathrm{H}$ ). The Codominant domain nucleates close to the hot spot at $\left(\mathrm{I}_{\mathrm{N}}\right.$ and $\mathrm{H}_{\mathrm{N}}$ ), the domain wall propagates and reaches the expansion pad at $\left(I_{P}\right.$ and $\left.H_{P}\right)$, it retracts from the expansion pad $\left(I_{R}\right.$ and $\mathrm{H}_{\mathrm{R}}$ ), and finally the Co dominant sublattice domain annihilates $\left(\mathrm{I}_{\mathrm{A}}\right.$ and $\left.\mathrm{H}_{\mathrm{A}}\right)$ at the original nucleation site. The evolution of those four fields is shown in Fig. 3. It is clear that, as 


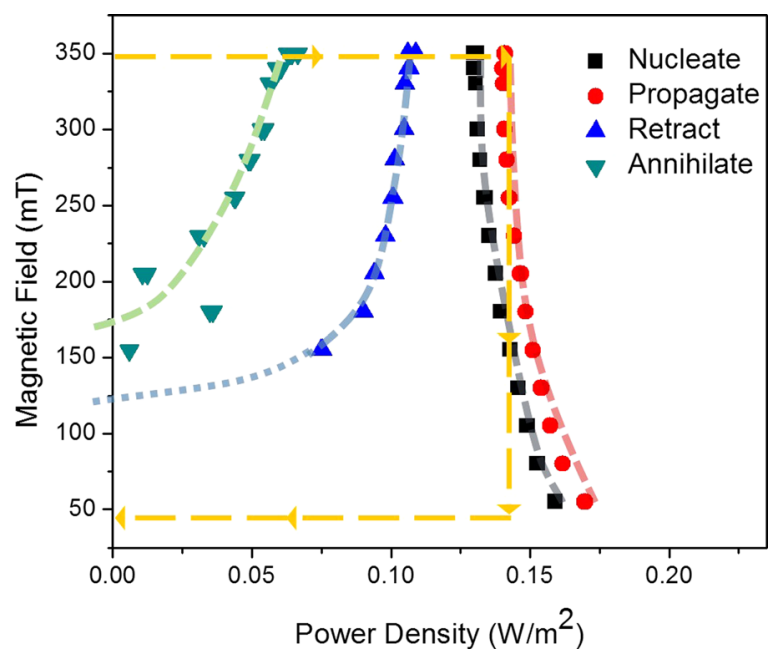

FIG. 3. Evolution of four different fields $\left(\mathrm{H}_{\mathrm{N}}, \mathrm{H}_{\mathrm{P}}, \mathrm{H}_{\mathrm{R}}\right.$, and $\left.\mathrm{H}_{\mathrm{A}}\right)$, respectively, the field at which the Cobalt dominant domain nucleates close to the hot spot (in black), the domain wall propagates and reaches the expansion pad (in red), the domain wall retracts from the expansion pad (in blue), and the Cobalt dominant domain annihilates (in green) as a function of the power dissipated in the wire-calculated across the entire sample as $I^{2} R$. Lines have been provided as a guide to the eye only. Each critical field has a corresponding required current. The yellow dashed line shows the current and field evolution that will create a domain wall between a Cobalt dominant domain and a Terbium dominant domain in the wire and then stabilize that domain at room temperature with zero applied field.

the sample approaches its compensation temperature, the critical fields $\left(H_{N}, H_{P}, H_{R}\right.$, and $\left.H_{A}\right)$ diverge as expected for ferrimagnetic systems.

From the evolution of these fields, it is possible to manipulate the DW with precision. For instance, for a given applied field $350 \mathrm{mT}$, if the current is increased upon reaching $\mathrm{I}_{\mathrm{N}}$ (for $\mathrm{H}=350 \mathrm{mT}$ ), the domain wall is nucleated and starts to propagate. During propagation, the domain wall moves and stops on specific sites along the wire suggesting domain wall pinning effects and thermally activated depinning. ${ }^{22}$ For a given current and field, the domain wall may be pinned in the middle of the wire. To stabilize the created domain wall, the retraction $\left(\mathrm{I}_{R}\right.$ and $\left.\mathrm{H}_{\mathrm{R}}\right)$ and annihilation $\left(\mathrm{I}_{\mathrm{A}}\right.$ and $\mathrm{H}_{\mathrm{A}}$ ) lines should not be crossed to avoid domain wall motion or annihilation. To do so, first, we need to reduce the field to zero while the temperature gradient is maintained and then reduce the injected current. This pathway in phase space is shown by the dotted line in Fig. 3. By reducing field but not changing the temperature, the picture in Fig. 4(a) is produced where the Co-dominant domain is stationary within the feature, and the thermal gradient is still active. Figure 4(b) is obtained when no current is flowing in the wire. The lack of a visual shift between the Kerr images in Figs. 4(a) and 4(b) indicates that the Co sublattice does not undergo rotation with the reduction of the applied heating current. However, with the cooling of the sample, the net film magnetization will rotate in the direction of the $\mathrm{Tb}$ sublattice as sketched in Figs. 4(c) and 4(d).

In summary, we are able to control the formation and propagation of domains within a model ferrimagnetic system near its compensation temperature. By crossing the compensation temperature while under applied field, a shift in sublattice dominance across $\mathrm{T}_{\text {comp }}$ is used to generate a domain wall within the material. This domain wall can be shifted

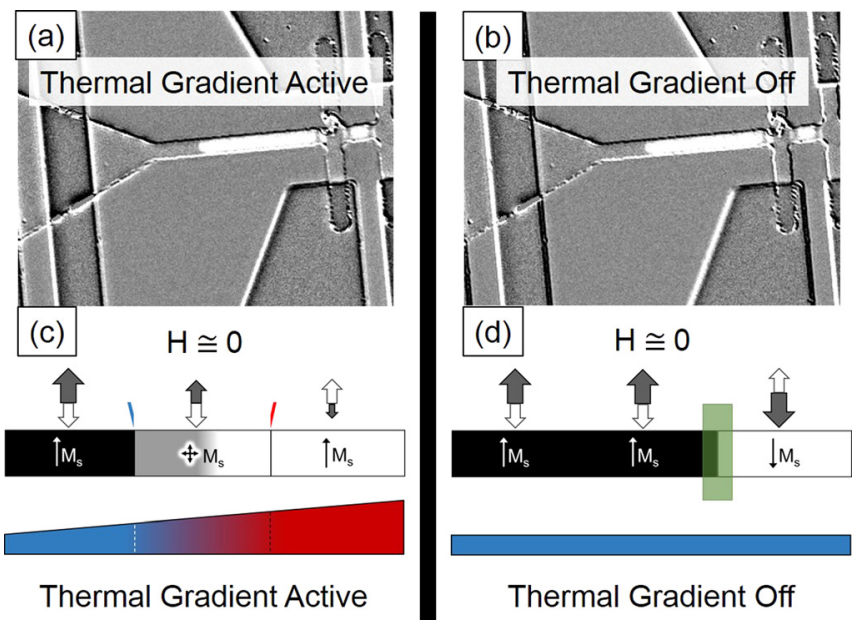

FIG. 4. Display of zero-field domain stabilization. (a) Kerr image of a domain wall created at $\mathrm{H}=350 \mathrm{mT}$ and for an injected current of $17 \mathrm{~mA}$ and then stabilized in zero field. (b) The thermal gradient has been removed. The expected magnetic configuration corresponding to (a) is sketched in (c), while the magnetic configuration for (b) is shown in (d).

and controlled by varying the applied joule heating current with the required temperatures for motion affected by the applied field. The use of a temperature gradient could be combined with polarized light for a better understanding of the role of heat and compensation in all optical switching experiments. ${ }^{9}$ Such effects could also be used on systems showing exchange bias behavior ${ }^{14,15}$ and GMR effects. ${ }^{16,17}$ Finally, synthetic ferrimagnetic materials ${ }^{9}$ which are compatible with application requirements could be studied to enhance the understanding of the behavior of such systems.

We would like to thank Raphael Marin and Austin Sanderson for their help with the experiment. This work was financially supported by the ANR Comag, the Partner University Fund (France embassy), FEDER France, the Region Lorraine, the Grand Nancy, and by the ANRT Labcom LSTMN. Robert Tolley received a Chateaubriand Fellowship to perform part of this work at Universite de Lorraine. Work at UCSD supported by the NSF Materials World Network Award 1312750.

${ }^{1}$ S. S. P. Parkin, M. Hayashi, and L. Thomas, "Magnetic domain-wall racetrack memory," Science 320(5873), 190-194 (2008).

${ }^{2}$ D. A. Allwood, G. Xiong, C. C. Faulkner, D. Atkinson, D. Petit, and R. P. Cowburn, "Magnetic domain-wall logic," Science 309, 1688 (2005).

${ }^{3} \mathrm{P}$. P. Freitas and L. Berger, "Observation of s-d exchange force between domain walls and electric current in very thin Permalloy films," J. Appl. Phys. 57, 1266 (1985).

${ }^{4}$ J. Grollier, A. Chanthbouala, R. Matsumoto, A. Anane, V. Cros, F. N. van Dau, and A. Fert, "Magnetic domain wall motion by spin transfer," C. R. Phys. 12, 309 (2011).

${ }^{5}$ N. Lei, T. Devolder, G. Agnus, P. Aubert, L. Daniel, J. Kim, W. Zhao, T. Trypiniotis, R. P. Cowburn, C. Chappert, D. Ravelosona, and P. Lecoeural, "Strain-controlled magnetic domain wall propagation in hybrid piezoelectric/ferromagnetic structures," Nat. Commun. 4, 1378 (2013).

${ }^{6}$ A. J. Schellekens, A. Van den Brink, J. H. Franken, H. J. M. Swagten, and B. Koopmans, "Electric-field control of domain wall motion in perpendicularly magnetized materials," Nat. Commun. 3, 847 (2012).

${ }^{7}$ J.-P. Tetienne, T. Hingant, J.-V. Kim, L. Herrera Diez, J.-P. Adam, K. Garcia, J.-F. Roch, S. Rohart, A. Thiaville, D. Ravelosona, and V. Jacques, "Nanoscale imaging and control of domain-wall hopping with a nitrogen-vacancy center microscope," Science 344, 1366-1369 (2014). 
${ }^{8}$ C. D. Stanciu, A. Tsukamoto, A. V. Kimel, F. Hansteen, A. Kirilyuk, A. Itoh, and T. Rasing, "Subpicosecond magnetization reversal across ferrimagnetic compensation points," Phys. Rev. Lett. 99, 217204 (2007).

${ }^{9}$ S. Mangin, M. Gottwald, C.-H. Lambert, D. Steil, L. Pang, M. Hehn, S. Alebrand, M. Cinchetti, G. Malinowski, Y. Fainman, M. Aeschlimann, and E. E. Fullerton, "Engineered materials for all-optical helicity-dependent magnetic switching," Nat. Mater. 13(3), 286-292 (2014).

${ }^{10}$ C.-H. Lambert, S. Mangin, B. S. D. Ch. S. Varaprasad, Y. K. Takahashi, M. Hehn, M. Cinchetti, G. Malinowski, K. Hono, Y. Fainman, M. Aeschlimann, and E. E. Fullerton, "All-optical control of ferromagnetic thin films and nanostructures," Science 345(6202), 1337 (2014).

${ }^{11}$ W. Jiang, P. Upadhyaya, Y. Fan, J. Zhao, M. Wang, L. Chang, M. Lang, K. L. Wong, M. Lewis, Y. Lin, J. Tang, S. Cherepov, X. Zhou, Y. Tserkovnyak, R. N. Schwartz, and K. L. Wang, "Direct imaging of thermally driven domain wall motion in magnetic insulators," Phys. Rev. Lett. 110, 177202 (2013).

${ }^{12} \mathrm{D}$. Hinzke and U. Nowak, "Domain wall motion by the magnonic spin Seebeck effect,” Phys. Rev. Lett. 107, 027205 (2011).

${ }^{13}$ A. Hrabec, N. T. Nam, S. Pizzini, and L. Ranno, "Magnetization reversal in composition-controlled $\mathrm{Gd}_{1-\mathrm{x}} \mathrm{Co}_{\mathrm{x}}$ ferrimagnetic films close to compensation composition," Appl. Phys. Lett. 99, 052507 (2011).

${ }^{14}$ S. Mangin, T. Hauet, P. Fischer, D. H. Kim, J. Kortright, K. Chesnel, E. Arenholz, and E. E. Fullerton, "Influence of interface exchange coupling in perpendicular anisotropy $[\mathrm{Pt} / \mathrm{Co}]_{50} / \mathrm{TbFe}$ bilayers," Phys. Rev. B 78, 024424 (2008).
${ }^{15}$ Y. Henry, S. Mangin, T. Hauet, and F. Montaigne, "Positive exchangebias induced by interface domain wall quenching in GdFe/TbFe films," Phys. Rev. B 73(13), 134420 (2006).

${ }^{16}$ C. Bellouard, H. D. Rapp, B. George, S. Mangin, G. Marchal, and J. C. Ousset, "Negative spin-valve effect in $\mathrm{Co}_{65} \mathrm{Fe}_{35} / \mathrm{Ag} /\left(\mathrm{Co}_{65} \mathrm{Fe}_{35}\right)_{50} \mathrm{Gd}_{50}$ trilayers," Phys. Rev. B 53(9), 5082 (1996).

${ }^{17}$ M. Gottwald, M. Hehn, F. Montaigne, D. Lacour, G. Lengaigne, S. Suire, and S. Mangin, "Magnetoresistive effects in perpendicularly magnetized Tb-Co alloy based thin films and spin valves," J. Appl. Phys. 111, 083904 (2012).

${ }^{18}$ T. Komine, K. Takahashi, A. Ooba, and R. Sugita, "Reduction of intrinsic critical current density for current-induced domain wall motion by using a ferrimagnetic nanowire with perpendicular magnetic anisotropy," J. Appl. Phys. 109, 07 D503 (2011).

${ }^{19}$ P. Hansen, C. Clausen, G. Much, M. Rosenkranz, and K. Witter, "Magnetic and magneto-optical properties of rare-earth transition-metal alloys containing Gd, Tb, Fe, Co,” J. Appl. Phys. 66, 756 (1989).

${ }^{20}$ A. Kunz, "Field induced domain wall collisions in thin magnetic nanowires,” Appl. Phys. Lett. 94, 132502 (2009).

${ }^{21}$ A. Zhukov, J. M. Blanco, A. Chizhik, M. Ipatov, V. Rodionova, and V. Zhukova, "Manipulation of domain wall dynamics in amorphous microwires through domain wall collision,” J. Appl. Phys. 114, 043910 (2013).

${ }^{22}$ C. Burrowes, D. Ravelosona, C. Chappert, S. Mangin, E. E. Fullerton, J. A. Katine, and B. D. Terris, "Role of pinning in current driven domain wall motion in wires with perpendicular anisotropy," Appl. Phys. Lett. 93, 172513 (2008). 